\title{
A Case Report of Mature Cystic Teratoma in a postmenopausal woman, a Rare Entity
}

\author{
Ahmed Hassan Abdelmoneim ${ }^{1}$, Adham Darweesh ${ }^{2 *}$, Abdelwahed Abougazia ${ }^{3}$, \\ Amal Said Mahan ${ }^{2}$, Amal Alobadli ${ }^{3}$
}

Received: 04 February 2021 / Accepted: 03 March 2021 / Published online: 20 July 2021

This article is published with open access at https://journal.astes.org.al

(C) The author(s) 2021. \& Copyright (C) 2021, the Albanian Society for Trauma and Emergency Surgery

(c) The Albanian Journal of Trauma and Emergency Surgery is an Open Access Journal. All articles are distributed under the terms of the Creative Commons Attribution Non-Commercial License: http://creativecommons.org/licenses/by-nc/4.0/) which permits unrestricted non-commercial use, distribution, and reproduction in any medium provided the original work is properly cited.

\begin{abstract}
Ovarian tumours are commonly seen in reproductive age group in women. However, when they occur at extremes of age, the suspicion of malignancy increases. The detection of dermoid cyst at 56 years of age is uncommon, hence proper imaging with CA-125 level monitoring is essential. Awareness of benign tumours or rare possibility of malignant transformation in such age is mandatory for surveillance management or planning the surgical procedure.
\end{abstract}

Keywords: postmenopausal woman, malignancy, mature cystic teratoma

\section{Background:}

The adnexal mass in a postmenopausal patient poses an important diagnostic and management dilemma for primary care providers and gynaecologists. Postmenopausal women are at a significantly increased risk of gynaecologic malignancy; yet even in this population most adnexal masses are benign [1]. Mature cystic teratoma is the most common type of ovarian germ cell tumour. It constitutes $20-30 \%$ of all ovarian tumours [2]. It is mostly seen in women younger than 40 years old and is seldom seen in postmenopausal women. They are bilateral in 10-15\%. Malignancy incidence is low (1-2\%) [3]. The incidence of malignancy in "adnexal mass increases with age, and approximately $30 \%$ are malignant in postmenopausal females [4]. It can have an unusual age presentation at 65 years with benign nature [5]. Pelvic ultrasound remains the mainstay for evaluation of adnex-

Original article, no submission or publication in advance or in parallel

\footnotetext{
* Corresponding author:

Dr. Adham Darweesh

$\bowtie$ adarweesh@hamad.qa

1 Hamad Medical corporation, Hamad General Hospital, Doha, Qatar \& Suez Canal University, EGYPT.

2 Hamad General Hospital, Doha, QATAR.

3 Department of radiology, Primary Health Care Corporation, Doha, QATAR.
}

al masses in premenopausal patients. If ultrasound findings are indeterminate, magnetic resonance imaging (MRI) is the next imaging modality of choice [6]. Tumour markers and imaging can help in the evaluation of adnexal mass in postmenopausal women [1]. For adnexal masses that are highly suspicious for cancer, women should be referred to a gynaecologic oncologist and facility for optimal care [1]. Optimal management of an asymptomatic adnexal mass allows continued surveillance, it is indicated if the suspicion of malignancy is low, but it has not been completely excluded. Surveillance usually includes serial pelvic ultrasounds and/ or measurement of serum tumour markers surveillance of women [7]. Surgery is recommended for any women with increasing morphologic complexity or a rising CA-125 [8].

\section{Case presentation:}

A 59 years old postmenopausal female presented with history of dysuria and frequency of micturition for few months. No history of renal disease or calculi. Recently complains of left iliac pain radiating to back with persistent frequency of micturition. On abdominal examination, it was soft, no organomegaly and no palpable masses felt with stable vital signs. A pelvi-abdominal ultrasound was requested and revealed a well-defined echogenic left adnexal mass measuring $7 X 7 \mathrm{~cm}$ with central hypo-echogenicity and fat-fluid level, no internal vascularity was seen by colour Doppler ultrasound, suggesting benign nature of the lesion. No ascites or lymph nodes were seen. The patient is referred to

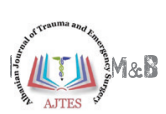


the emergency for gynaecological assessment and further imaging recommendation. MRI was highly recommended to exclude neoplastic lesions. CA-125 (tumour marker) $=$ 9.7 U/ml (high normal=35 U/ml.). Gynaecologic Cytology report (cervical liquid specimen) was negative for intraepithelial lesion or malignancy. MRI pelvis showed a large cystic lesion of $9 \times 7 \times 7 \mathrm{~cm}$ in the left adnexa with the left ovary not seen separately. (figure 1-7). No enhancement is seen with fat intensity in all sequences suggesting left ovarian dermoid (mature cystic teratoma). The patient went through a laparotomy (total abdominal hysterectomy with bilateral salpingo-oophorectomy, omental biopsy and peritoneal wash that revealed histopathological evidence of a left ovarian mature cystic teratoma.

\section{Discussion:}

An "adnexal mass" is a common specimen encountered in the daily pathology practice. Although most are benign, the incidence of malignancy increases with age, and approximately $30 \%$ are malignant in postmenopausal females [6]. Gynaecologists must differentiate between benign and malignant adnexal masses. The evidence supporting to the specialty care of a gynaecologic oncologist based on the physical examination, imaging studies, family history, and CA 125 determination [9].

In our case, we started with transvaginal Ultrasound and the lesion was indeterminate, so MRI was recommended, and the patient was referred. The tertiary care hospital followed the guidelines for referral of patient with adnexal mass study done by Gostout BS \& Brewer MA on 2006 [9] and CA-125 was done, followed by MRI. According to a study that mentioned RMI (The score incorporates the patient's menopausal status (M), ultrasound features of the lesion (U) was the best method for discrimination between BOTs (borderline ovarian tumours) and benign adnexal masses and was more accurate than the other parameters [10]. In our case, the RMI $(\mathrm{RMI}=$ $\mathrm{U} \times \mathrm{M} \times \mathrm{CA}-125(\mathrm{U} / \mathrm{ml})=0$ X 3 X $9.7=0)$ results are $(0)<25$ and our patient is categorized as "low risk" showing benign looking in all imaging modalities and CA-125 is low normal with no evidence of malignancies or metastases. The management of an adnexal mass depends upon the type of mass, urgency of the presentation, and degree of suspicion that the mass is malignant [7]. Low risk patients as in our study, for most women with a benign looking ovarian lesion and no other findings suggestive of malignancy, the study suggested surveillance rather than surgery [7]. However, the larger the mass, the less certain the sonologist can be about the content of the mass. Finally, a certain subset of these masses will continue to grow so one would rather remove them when they are within range for laparoscopic management [7]. In our case, the patient started to be symptomatic in the last few months with a large size of the ovarian mass so, the gynaecology oncologist decided to go through a laparotomy: total abdominal hysterectomy, bilateral salpingo-oophorectomy, omental biopsy and peritoneal wash and the pathology report revealed a left ovarian mature cystic teratoma.

\section{Conclusion:}

Mature cystic teratoma is generally seen in reproductive age group; however, rare cases may occur in prepuberty age group to women in postmenopausal age group. Although the rate of malignant transformation is only $1 \%$ in patients with mature cystic teratoma, this may be more commonly seen in those patients with extremes of age.

\section{Declarations:}

\section{Competing interests}

The authors declare that they have no competing interests.

\section{Ethical Considerations:}

Informed written consent from the patient included in this study was taken.

\section{Consent for publication:}

All authors read and approved the final manuscript.

\section{Availability of data and material:}

The data that support the findings of this study are available on request from the corresponding author.

\section{References:}

1. Rauh-Hain JA, Melamed A, Buskwofie A, Schorge JO. Adnexal mass in the postmenopausal patient. Clinical Obstetrics and Gynecology. 2015 Mar 2;58(1):53-65.

2. Ayhan A, Bukulmez O, Genc C, Karamursel BS, Ayhan A. Mature cystic teratomas of the ovary: case series from one institution over 34 years. Eur J Obstet Gynecol Reprod Biol. 2000; 88:153-7.

3. Ozgur T, Atik E, Silfeler DB, Toprak S: Mature cystic teratomas in our series with review of literature and retrospective analysis. Arch Gynecol Obstet. 2012, 285:1099-1101. 438.

4. Bennett, Jennifer \& Oliva, Esther. Pathology of the Adnexal Mass. Clinical obstetrics and gynecology. March 2015;58(1): 3-27.

5. Inkollu S, Nelavelli A, Merugu SM. An unusual age presentation of mature cystic teratoma: a case report. Int J Reprod Contracept Obstet Gynecol 2015; 4:1234-6.

6. Hall TR, Randall TC. Adnexal Masses in the Premenopausal Patient. Clinical Obstetrics and Gynecology: March 2015 Volume 58 - Issue 1 - p 47-52.

7. Muto MG. Management of an adnexal mass: In: UpToDate, Post TW (Ed), UpToDate, Waltham, MA. (Accessed on July 15, 2020)

8. Iyer VR, Lee SI. MRI, CT, and PET/CT for ovarian cancer detection and adnexal lesion characterization. AJR. American journal of roentgenology 2010. 194. 311-21.

9. Gostout BS, Brewer MA. Guidelines for referral of the patient with an adnexal mass. Clinical Obstetrics and Gynecology. 2006 Sep;49(3):448-458.

10. Alanbay I, Aktürk E, Coksuer H, Ercan M, Karasahin K, Dede $\mathrm{M}$, et al. Comparison of risk of malignancy index (RMI), CA125, CA 19-9, ultrasound score, and menopausal status in borderline ovarian tumour. Gynaecological endocrinology: the official journal of the International Society of Gynaecological Endocrinology. 2011;28. 478-82. 10.3109/09513590.2011.633663. 


\section{Figures}

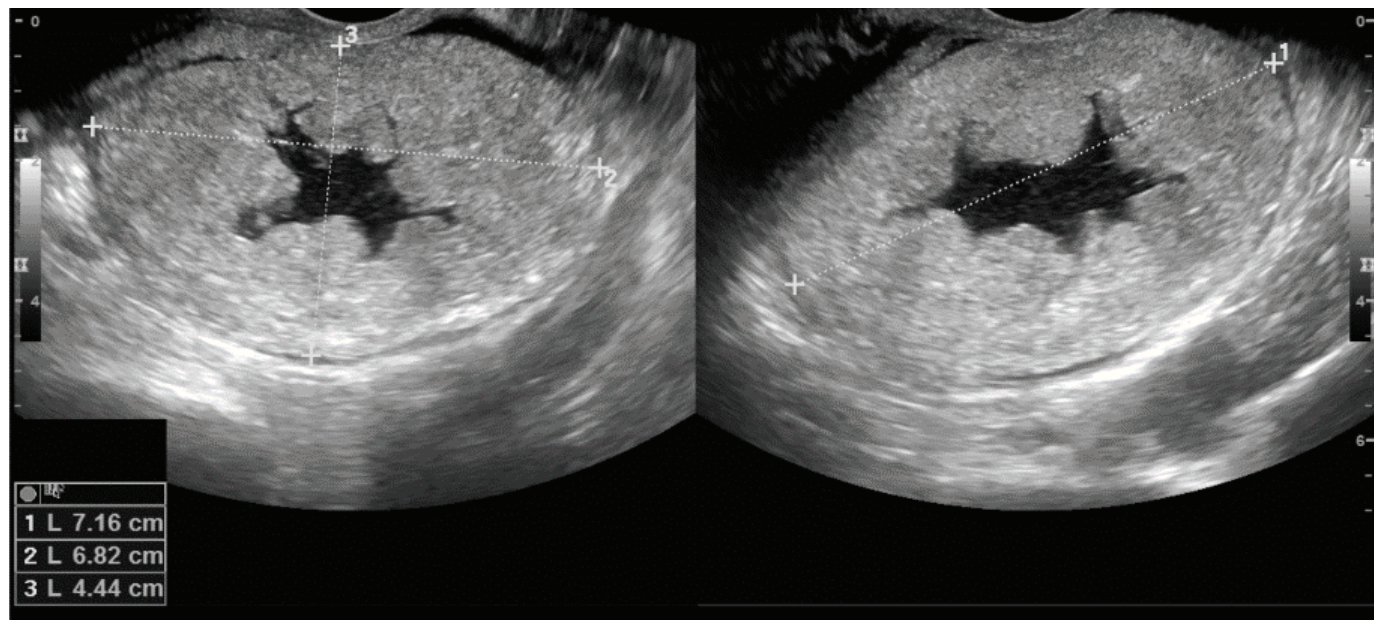

Figure 1: Pelvic ultrasound revealed a well-defined mass with echogenic peripheral part and anechoic central part.

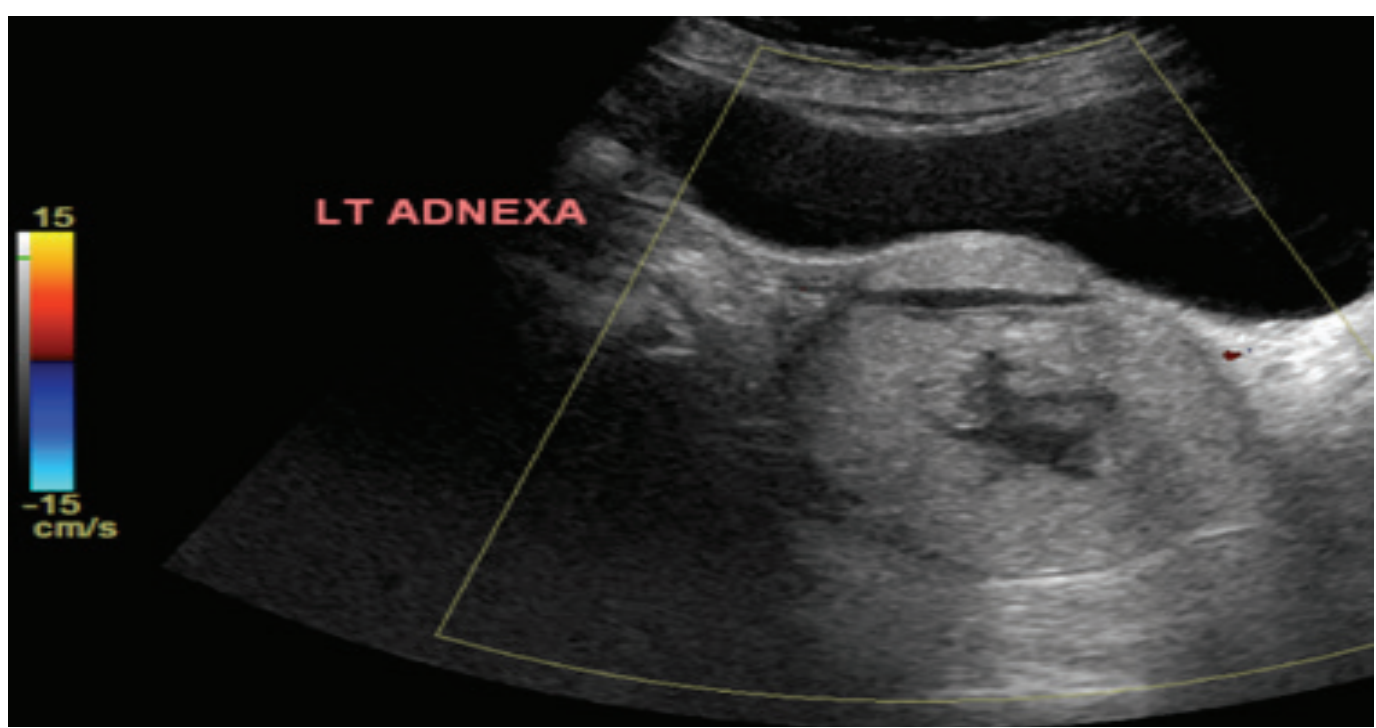

Figure 2: Coloured Doppler of the same lesion shows no internal vascularity.

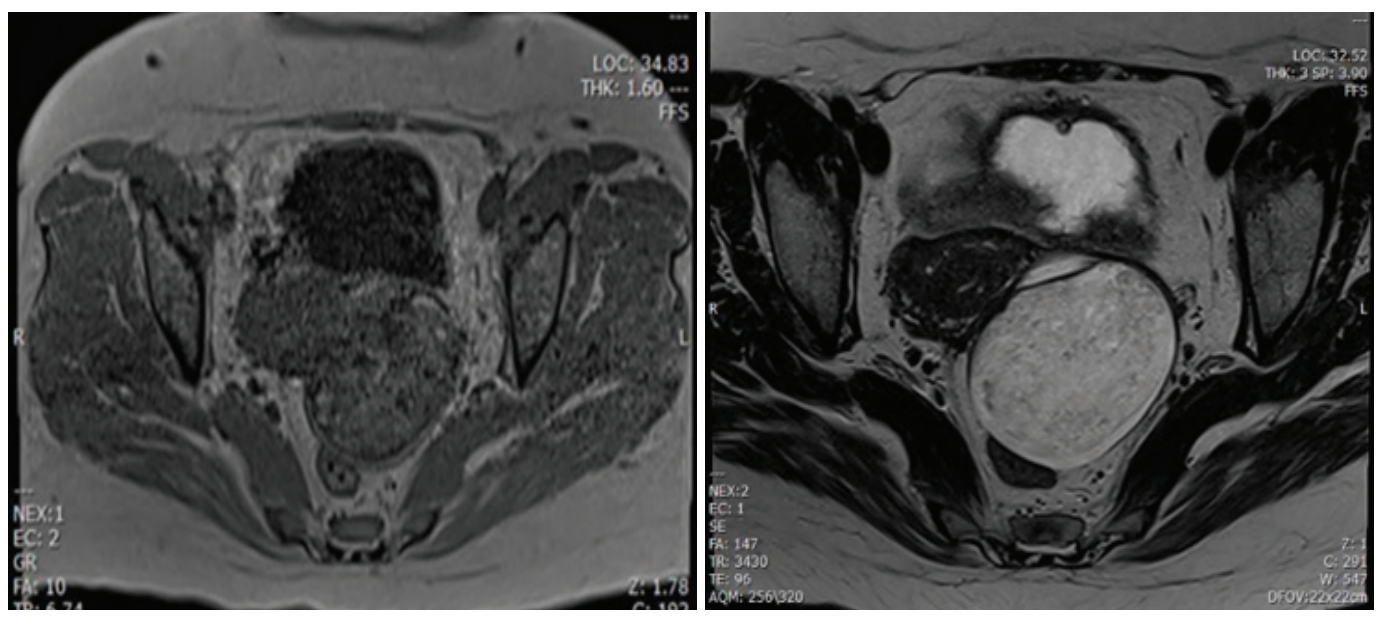

Figure 3: T1 WI shows heterogeneous intermediate signals and T2 WI shows intermediate to high signals. 


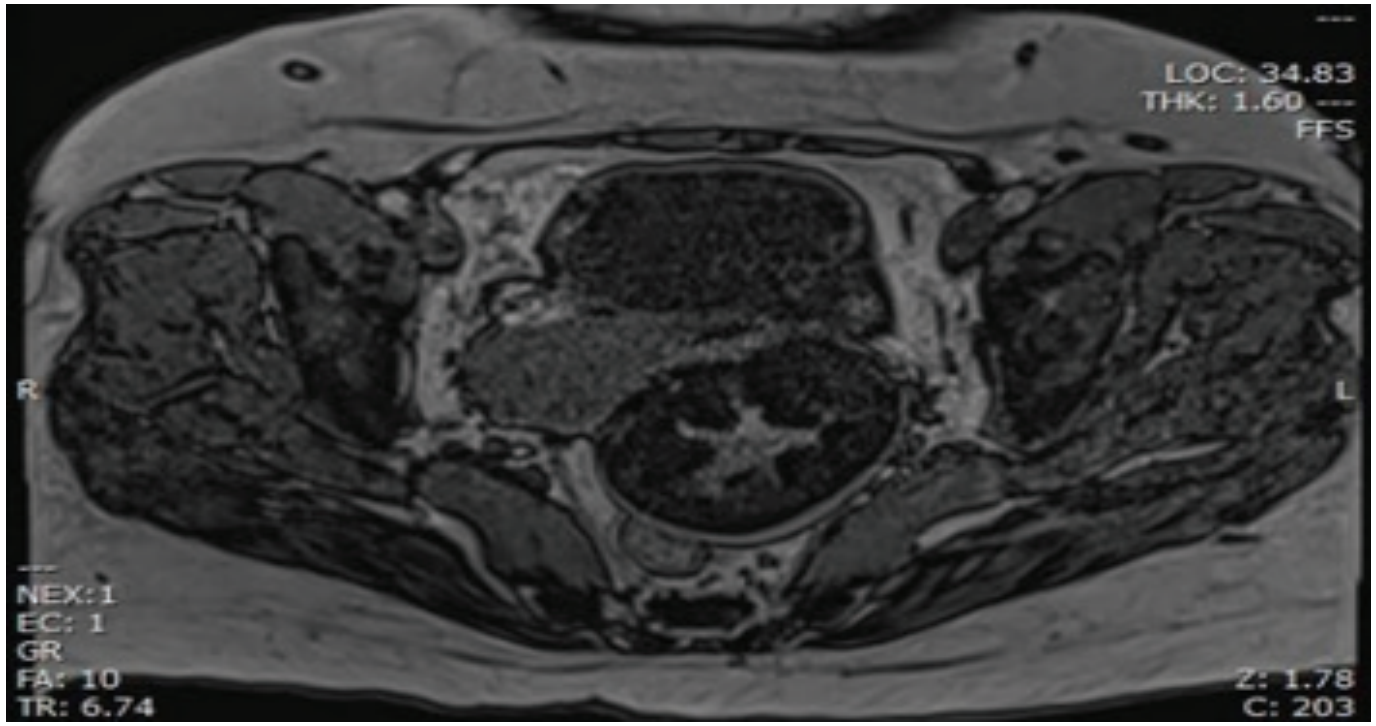

Figure 4: Out of phase shows drop of signals, denoting microscopic fat.

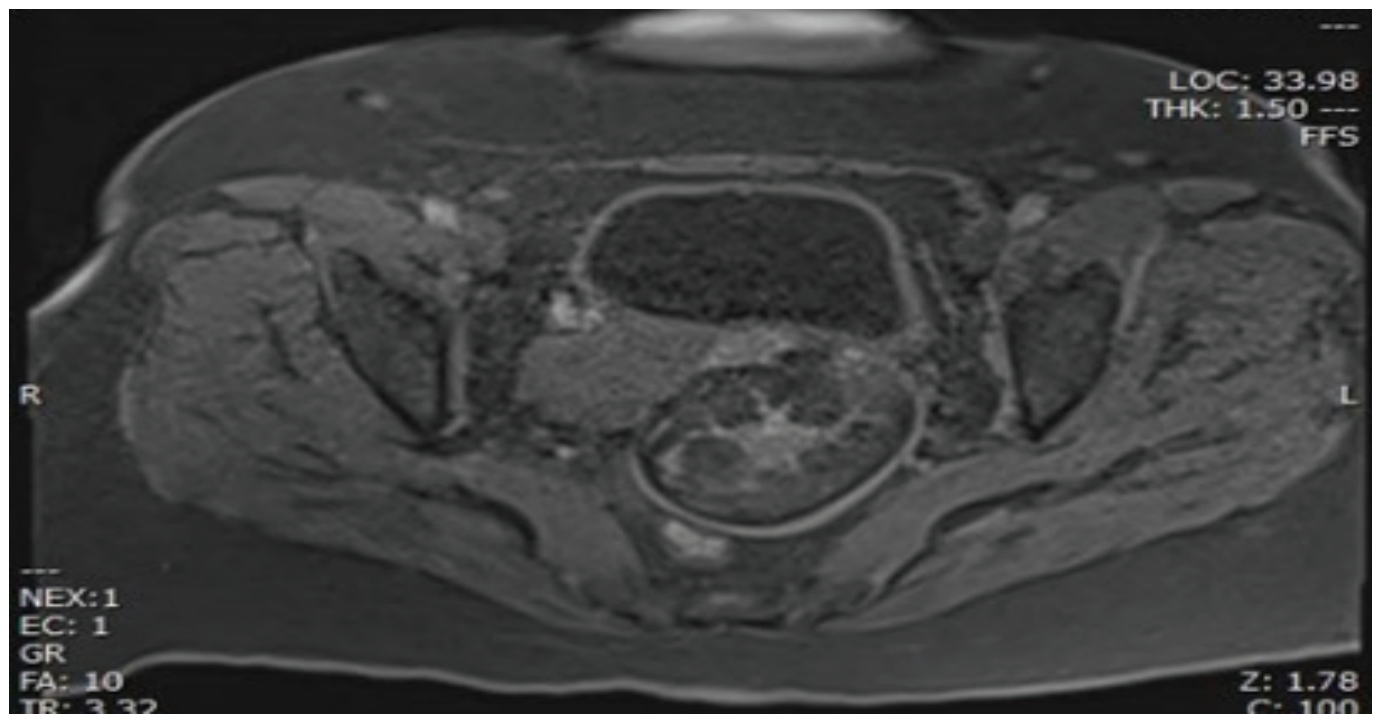

Figure 5: T1 FatSat WI shows darker signals, in compatible with macroscopic fatty contents.

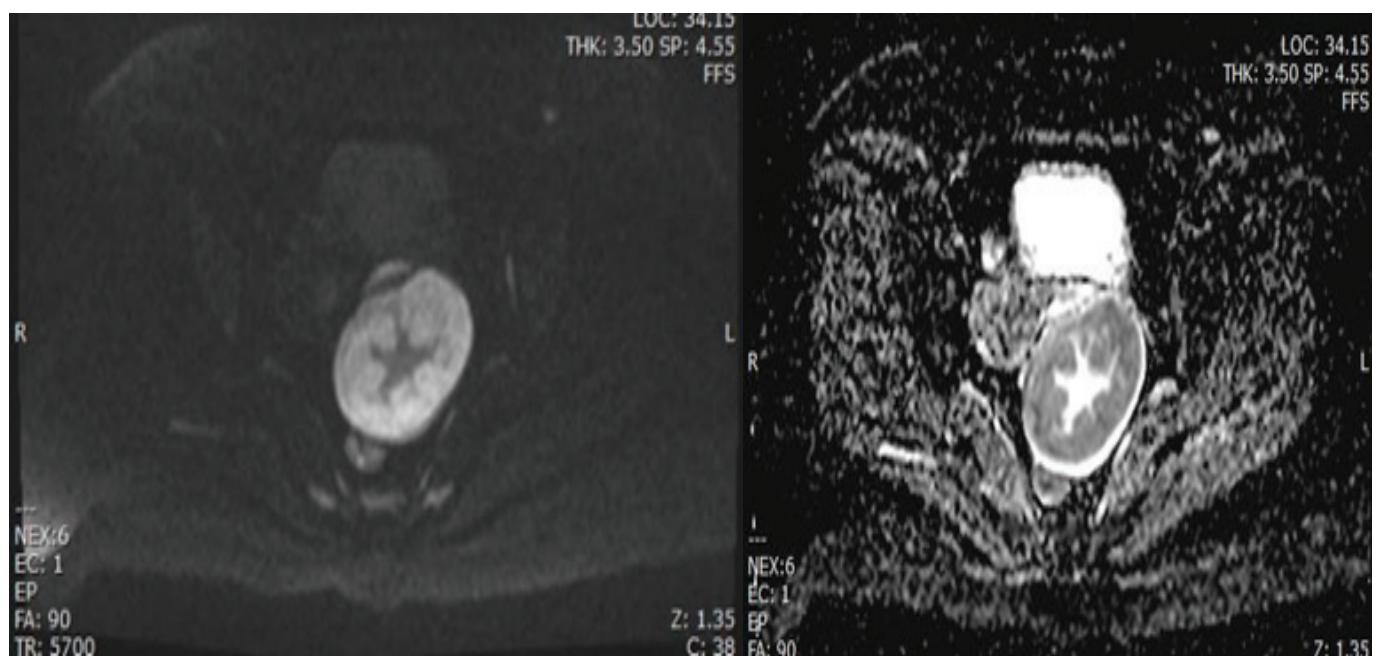

Figure 6: DWI and ADC of the mass shows restricted diffusion. 


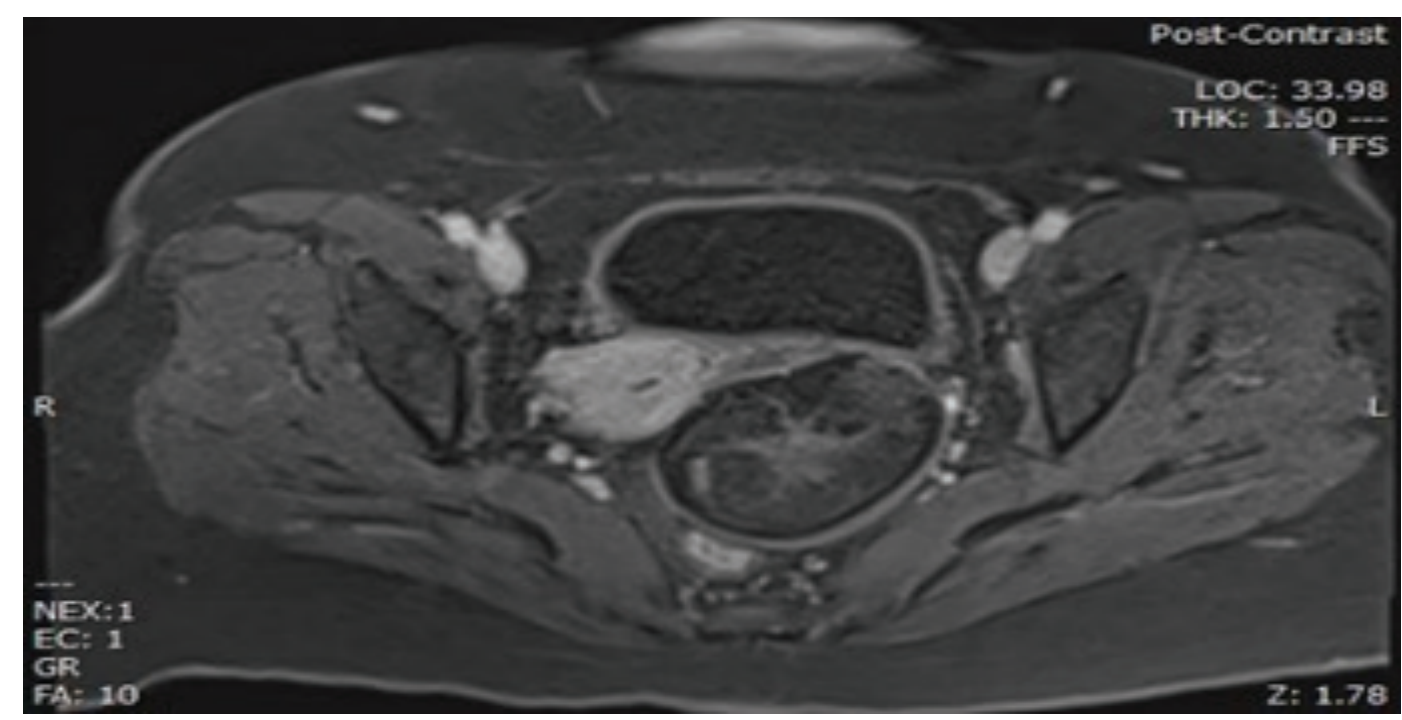

Figure 7: T1 FatSat with contrast shows no enhancement of the mass. 